\section{A situação de aleitamento materno de crianças atendidas em creches da Secretaria da Assistência Social do município de São Paulo - região Freguesia do Ó}

\section{Breast-feeding in children in day care centers of the Social Welfare Department of the Municipality of São Paulo - Freguesia do Ó area}

Mônica G. N. Spinelli 1

Edna H. Sesoko 2

José Maria P. Souza 3

Sônia B. de Souza 4

1-2 Secretaria da Assistência Social da Prefeitura Municipal de São Paulo. Rua Chico de Paula, 224. Frequesia do Ó. São Paulo, São Paulo, Brasil. CEP: 02.926-000

3 Departamento de Epidemiologia da Faculdade de Saúde Pública da Universidade de São Paulo

${ }^{4}$ Departamento de Nutrição da Faculdade de Saúde Pública da Universidade de São Paulo

\begin{abstract}
Objectives: to evaluate breast-feeding in children, under 18 months old in day care centers and identify the variables associated to the weaning process.

Methods: two hundred and thirty five mothers of under -18 months' old infants, in day care centers of the Social Welfare Department of the Municipality of São Paulo, São Paulo, Brazil (Freguesia do Ó area) were interviewed in February 2000. Association of breast-feeding to maternal age and education level, delivery type and birthweight was made. The $\chi 2$ test was conducted to compare the proportions when factors associated to precocious weaning were studied. Relative risk $(R R)$ was calculated for prevalence. The survival analysis was used to calculate breast-feeding duration.

Results: initial breast-feeding prevalence was $92,7 \%$ and the mean duration was 5,6 months. 80,4\% of the children were breast-feeding during the first month of life and $50 \%$ were weaned by the end of the forth month.

Conclusions: none of the variables studied had the requirements to enable inclusion in a multivariate model and did not show any association to weaning. Key words Breast-feeding, Weaning, Child day care centers
\end{abstract}

\section{Resumo}

Objetivos: avaliar a situação de aleitamento materno entre crianças menores de dezoito meses que freqüentam creches e identificar as variáveis associadas ao desmame.

Métodos: foram entrevistadas, no mês de fevereiro de 2000, mães de 235 crianças menores de dezoito meses que freqüentavam as creches ligadas à Secretaria da Assistência Social do Município de São Paulo, São Paulo, Brasil, (região Freguesia do Ó). Foi investigado a associação do aleitamento com idade e escolaridade maternas, tipo de parto e peso ao nascer das crianças. O teste $\chi 2$ foi empregado para comparação das proporções quando se estudaram os fatores associados ao desmame precoce. Foi calculado o risco relativo $(R R)$ para as prevalências. Para a análise da duração do aleitamento materno utilizou-se a técnica da tábua de vida.

Resultados: a prevalência de amamentação das crianças que foram amamentadas alguma vez por suas mães foi 92,7\% com uma duração media de 5,6 meses. $80,4 \%$ das crianças foram amamentadas no primeiro mês de vida e no final do quarto mês, $50 \%$ já se encontravam desmamadas.

Conclusões: nenhuma das variáveis estudadas apresentou condições para entrada em um modelo multivariado; também não mostraram associação com o desmame.

Palavras-chave Aleitamento materno, Desmame, Creches 


\section{Introdução}

O leite materno é inquestionavelmente o melhor alimento para o recém-nascido, por sua composição nutricionalmente equilibrada, temperatura adequada, por ser estéril, ter composição variável de acordo com a maturação das mamas e da capacidade gástrica da criança, possibilitar uma relação mãe/filho harmoniosa e praticamente não propiciar reações alérgicas.1,2 Além disso tem vantagens de ordem econômica. ${ }^{3}$ Garante assim a alimentação ideal para o bebê e contribui para seu crescimento e desenvolvimento saudável, reduzindo a incidência e a severidade de enfermidades.4,5 A World Health Organization (WHO) 6 preconiza a amamentação de forma exclusiva até, pelo menos, o quarto mês de vida. O equilíbrio de seus nutrientes possibilita à criança alcançar o máximo de desenvolvimento do potencial genético. ${ }^{7}$

Há uma substancial redução de custos para os serviços de saúde quando a criança recebe aleitamento exclusivo nos três primeiros meses, decorrente da diminuição da morbidade no primeiro ano de vida. ${ }^{8}$ Há evidências de forte associação entre aleitamento materno e menor risco de infecções do trato respiratório baixo bem como entre tempo de amamentação e esse tipo de enfermidade. ${ }^{9}$ Este fator é de particular importância para a criança que freqüenta creche, desde que aí tende a ocorrer um risco maior de desenvolvimento de doenças infecciosas e de morbidade por doenças respiratórias, devido à rapidez com que determinados patógenos são transmitidos em comunidades fechadas.

Assim, o estudo das variáveis que possam interferir negativamente na prática de amamentação permite que se criem estratégias para minimizar esse efeito.

O presente trabalho propôs-se avaliar a situação de aleitamento materno entre crianças menores de 18 meses que freqüentam creches e identificar as variáveis associadas ao desmame.

\section{Métodos}

Foram entrevistadas, durante o mês de fevereiro de 2000, mães de 235 crianças menores de 18 meses, usuárias de 12 creches da Secretaria Municipal da Assistência Social, região Freguesia do Ó, município de São Paulo, São Paulo, Brasil. Esse número de crianças representa aproximadamente $35 \%$ do atendimento dessa faixa etária, nessa região. $\mathrm{O}$ critério para participação na pesquisa foi a aceitação do projeto pelas diretoras das creches e o consentimento esclarecido das mães; também foi escolhida a faixa etária menor de dezoito meses para garantir uma maior fidelidade das respostas às perguntas retrospectivas.

O levantamento foi feito por auxiliares de enfermagem que trabalham nas creches, sob orientação das nutricionistas responsáveis pela supervisão, após esclarecimento consentido das mães, utilizando questionário estruturado com a maioria das questões fechadas (previamente codificadas) e algumas questões abertas.

Foi escolhida a faixa etária menor de dezoito meses para garantir uma maior fidelidade das respostas nas perguntas retrospectivas.

Foram estudadas as seguintes variáveis: sexo e idade da criança, tipo de parto, peso ao nascer, idade e escolaridade materna, paridade e orientação de aleitamento materno.

Para análise das variáveis utilizaram-se as seguintes classificações:

- Tipo de parto - normal ou cirúrgico;

- Idade materna $₹ 20$ anos - mãe adolescente; 20 - 30 anos; > 30 anos;

- Peso ao nascer $₹ 2.500 \mathrm{~g}$ - baixo peso; 2.500 2.999 - peso deficiente; $\geq 3.000$ - peso normal. Para efeito de comparação de dados com outros estudos, foi utilizada a categorização estabelecida por Puffer e Serrano. 10

O teste de $\chi^{2}$ foi empregado para comparação das proporções quando se estudaram os fatores associados ao desmame precoce admitindo-se um erro alfa de 5\%. Foi calculado o risco relativo (RR) para a razão das prevalências. ${ }^{11,12}$ Para processamento e análise dos dados foram utilizados os programas Epi-info versão 613e Stata versão 6.14

Para a análise da duração do aleitamento materno utilizou-se a técnica da tábua de vida, conforme modelo de Cox. Considerou-se o fato de receber leite materno como critério de aferição. ${ }^{14,15} \mathrm{Na}$ análise da duração do aleitamento materno o evento final foi a interrupção definitiva da amamentação. A terminologia aleitamento materno empregada neste texto refere-se ao recebimento de leite materno independente da criança receber concomitantemente água ou outro alimento.

\section{Resultados}

Observou-se uma distribuição homogênea em relação ao sexo com ligeira predominância do sexo masculino.

A idade das crianças variou de dois meses a 18 meses sendo a média 11,9 meses $(\mathrm{dp}=4,03)$.

Em relação ao acompanhamento pediátrico $88,1 \%$ das crianças freqüentavam regularmente o 
consultório do pediatra e $18,7 \%$ tinham consulta somente em caso de urgência. Os pediatras foram responsáveis por $33,6 \%$ das orientações de aleitamento.

Conforme a Tabela 1 a maior parte das mães recebeu orientação de aleitamento materno na maternidade.

A Tabela 2 mostra que $70,2 \%$ das crianças que freqüentavam as creches já se encontravam desmamadas. Verifica-se também que, do total de crianças, 92,7\% receberam leite materno exclusivo ou combinado com outro alimento ou água em algum período da vida, enquanto $7,3 \%$ nunca foram amamentadas.
Para o total das crianças a duração mediana do aleitamento foi 5,6 meses. 7\% das mães, que tinham maior disponibilidade de horário, amamentaram suas crianças na creche.

A Tabela 3 mostra os motivos referidos pelas mães para interrupção da amamentação. Observa-se que "Recusa da criança", o "Leite secou" e o "Trabalho" foram os três principais motivos alegados pelas mães para não amamentar ou interromper a amamentação, atingindo, cada uma delas, freqüências da ordem de 25 a $28 \%$.
Tabela 1

Distribuição das mães segundo orientação de aleitamento.

\begin{tabular}{lc}
\hline \multicolumn{1}{c}{ Local de orientação } & n \\
\hline Pré-natal & 47 \\
Maternidade & 130 \\
Consulta pediátrica & 80 \\
Outros & 22 \\
Total & $279 *$ \\
\hline
\end{tabular}

* Não se refere a $100 \%$ pois algumas mães referiram mais de uma resposta.
Tabela 3

Motivos alegados pelas mães para não amamentar ou interrupção da amamentação.

\begin{tabular}{lrr}
\hline \multicolumn{1}{c}{ Motivo } & $\mathbf{n}$ & $\%$ \\
\hline Recusa da criança & 51 & 28,4 \\
Leite secou & 49 & 27,3 \\
Trabalho & 46 & 25,7 \\
Mãe não teve leite & 8 & 4,5 \\
Doença da mãe & 7 & 3,9 \\
Internação/doença da criança & 5 & 2,8 \\
Pouco leite & 3 & 1,7 \\
Introdução de mamadeira & 3 & 1,7 \\
Outros motivos & 7 & 3,9 \\
Total & 179 & 100,0 \\
\hline
\end{tabular}

Distribuição das crianças em relação ao aleitamento e faixa etária.

\begin{tabular}{|c|c|c|c|c|c|c|c|c|}
\hline \multirow[b]{3}{*}{ Situação de Aleitamento } & \multicolumn{8}{|c|}{ Faixa etária } \\
\hline & \multicolumn{2}{|c|}{$<6$ meses } & \multicolumn{2}{|c|}{ 6-12 meses } & \multicolumn{2}{|c|}{$>12$ meses } & \multicolumn{2}{|c|}{ Total } \\
\hline & $\mathrm{n}$ & $\%$ & $\mathrm{n}$ & $\%$ & $\mathrm{n}$ & $\%$ & $\mathrm{n}$ & $\%$ \\
\hline Estavam em aleitamento & 15 & 78,9 & 22 & 22,9 & 16 & 13,3 & 53 & 22,5 \\
\hline Estavam desmamadas & 3 & 15,8 & 62 & 64,6 & 100 & 83,3 & 165 & 70,2 \\
\hline Nunca amamentadas & 1 & 5,3 & 12 & 12,5 & 4 & 3,4 & 17 & 7,3 \\
\hline Total & 19 & 100,0 & 96 & 100,0 & 120 & 100,0 & 235 & 100,0 \\
\hline
\end{tabular}


Conforme se pode verificar na Tabela 4 não houve associação entre aleitamento e idade materna $(\mathrm{p}=$ $0,42)$, escolaridade materna $(\mathrm{p}=0,20)$ ou tipo de parto $(\mathrm{p}=0,80)$. Observa-se ainda que 153 mães $(66 \%)$ tinham escolaridade superior à $5^{\text {a }}$ série, 25 (10,8\%) eram menores de 20 anos e 149 (64\%) estavam na faixa dos 20 a 30 anos. A proporção de partos normais foi de $62 \%$ (139 partos). A Tabela 5 mostra que também não houve associação de condições de aleitamento com baixo peso ao nascer ( $\mathrm{p}=0,20)$. Observa-se ainda que $18(7,9 \%)$ crianças tinham baixo peso e $147(64,4 \%) 3.000 \mathrm{~g}$ ou mais.

A análise das Tabelas 4 e 5 revela, ademais, que nenhuma das variações apresenta condições para entrada no modelo multivariado. 14

Tabela 4

Distribuição das crianças conforme o aleitamento em relação às variáveis maternas.

\begin{tabular}{|c|c|c|c|c|c|c|c|c|c|}
\hline \multicolumn{10}{|c|}{ Aleitamento } \\
\hline & & & & & & & & & \\
\hline & & $\mathrm{n}$ & $\%$ & $\mathrm{n}$ & $\%$ & Total & RR & $\chi^{2}$ & $p$ \\
\hline \multirow[t]{3}{*}{ Idade materna (anos)* } & $<20$ & 23 & 92,0 & 2 & 8,0 & 25 & 1,0 & & \\
\hline & 20 a 30 & 136 & 91,3 & 13 & 8,7 & 149 & 0,99 & 1,73 & 0,42 \\
\hline & $>30$ & 56 & 96,6 & 2 & 3,4 & 58 & 1,05 & & \\
\hline Escolaridade & até $4^{a}$ série & 74 & 94,9 & 4 & 5,1 & 78 & 1,00 & & \\
\hline \multirow[t]{2}{*}{ materna** } & $5^{a}$ a $8^{a}$ & 91 & 89,2 & 11 & 10,8 & 102 & 0,94 & 3,21 & 0,20 \\
\hline & $2^{\circ} \mathrm{grau}$ e mais & 49 & 96,2 & 2 & 3,9 & 51 & 0,10 & & \\
\hline \multirow[t]{2}{*}{ Tipo de parto*** } & Normal & 129 & 92,8 & 10 & 7,2 & 139 & 1,00 & & \\
\hline & cirúrgico & 47 & 91,9 & 7 & 8,1 & 54 & 0,98 & 0,07 & 0,80 \\
\hline
\end{tabular}

* Sem informação para três crianças, * sem informação para quatro crianças, *** sem informação para dez crianças, RR = Risco relativo

Tabela 5

Distribuição das crianças conforme o aleitamento em relação ao peso ao nascer.

\begin{tabular}{|c|c|c|c|c|c|c|c|c|c|}
\hline \multicolumn{10}{|c|}{ Aleitamento } \\
\hline & & \multicolumn{2}{|c|}{ Sim } & \multicolumn{2}{|c|}{ Não } & \multirow{2}{*}{ Total } & \multirow{2}{*}{$\mathrm{RR}$} & \multirow{2}{*}{$\chi^{2}$} & \multirow{2}{*}{$\mathrm{p}$} \\
\hline & & $\mathrm{n}$ & $\%$ & $\mathrm{n}$ & $\%$ & & & & \\
\hline Peso ao nascer & $<2.500$ & 17 & 94,4 & 1 & 5,6 & 18 & 1,0 & & \\
\hline \multirow[t]{2}{*}{ (gramas)* } & $2.500-2.999$ & 61 & 96,8 & 2 & 3,2 & 63 & 1,03 & 2,24 & 0,20 \\
\hline & $>3.000$ & 134 & 91,2 & 13 & 8,8 & 147 & 0,97 & & \\
\hline
\end{tabular}

* Sem informação para sete crianças, RR = Risco relativo 
A curva de sobrevida das crianças amamentadas (Figura 1), segundo o modelo de Cox, indica que $50 \%$ das crianças já estavam desmamadas ao final do quarto mês de vida.

\section{Figura 1}

Curva de sobrevida das crianças amamentadas.

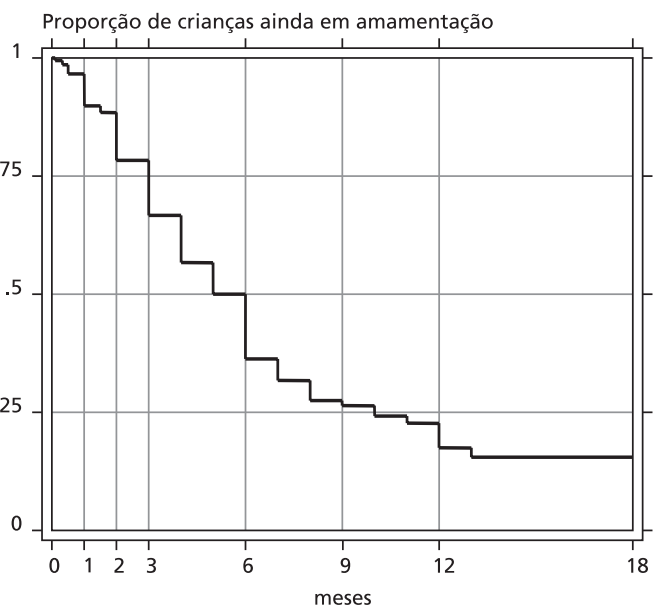

\section{Discussão}

A prevalência de aleitamento materno das crianças que foram amamentadas pelo menos uma vez por sua mãe, foi semelhante à encontrada em vários outros estudos $1,16-18$ que mostraram valores de $92,9 \%, 92,8 \%$, $92,3 \%$ e $93,5 \%$. A duração media do aleitamento foi 5,6 meses, inferior portanto aos sete meses para amamentação conforme referido pela Pesquisa Nacional sobre Demografia e Saúde realizada em várias regiões do País. 19

As creches aqui estudadas, caso a mãe tivesse disponibilidade e desejo de amamentar, procuravam contribuir para o incentivo ao aleitamento materno, propiciando condições, tais como local e horários especiais. Apesar desta disponibilidade, havia pouca viabilidade para tal, considerando-se que: 1) a maior parte das mães trabalhavam;2) poucas mães tinham disponibilidade de horário para amamentar.

Observou-se que a maternidade foi o principal local de orientação de aleitamento materno, mesmo assim com uma cobertura parcial de 55,3\%. Apesar do número expressivo de crianças em acompa- nhamento regular pelo pediatra, apenas $1 / 3$ das mães referiram ter recebido orientação de aleitamento nesta ocasião.

A família e as pessoas do círculo de amizade, identificados neste trabalho como "Outros" e que, eventualmente, orientam e compartilham experiências de amamentação, parecem ter pouca interferência em relação à orientação de aleitamento materno. Apesar da maioria das mães ter referido algum estímulo à amamentação, estes são ainda insuficientes, sendo portanto o comportamento em relação a amamentação, ainda insatisfatório.

Com respeito à condição de aleitamento, observou-se que a maior parte das menores de seis meses encontrava-se em aleitamento materno, o que sugere que, embora a mãe não compareça à creche para amamentar, ainda mantem o aleitamento em algum período do dia para as crianças menores. Após o sexto mês há um declínio acentuado do desmame.

Os principais motivos alegados para a interrupção da amamentação foram a "Recusa da criança", a "Diminuição ou término do leite" e o "Trabalho", o que é similar aos evidenciados em outros estudos.1,2,4,18,20 Apesar da alta porcentagem de mães que deixavam a criança na creche para ir ao trabalho, este aparece apenas como terceira causa de desmame.

Embora a condição necessária para matrícula da criança na creche, seja uma renda familiar inferior a três salários mínimos - valores aos quais poder-se-ia atribuir como motivo para uma baixa escolaridade observou-se que dois terços das mães tinham um nível de escolaridade superior à 5 a série. Diferentemente dos nossos resultados, entretanto, Barber21 e Marchioni22 encontraram associação positiva entre aleitamento e escolaridade materna.

Estudos anteriores mostraram também uma correlação entre tempo de desmame e introdução de alimentos sólidos com idade materna. 23,24 Candeias 23 observou que mulheres com idade de 29 anos ou menos apresentavam maior risco de desmame precoce. O presente trabalho porém não reproduziu estes achados.

Há estudos mostrando que mães com experiências anteriores de amamentação, tendem a repetir a experiência e amamentar por mais tempo, caso tenham disponibilidade, 25 o que suporta os resultados encontrados no presente

Embora o nosso estudo tenha mostrado que 7,9\% das crianças tinham peso baixo ao nascer, não revelou associação desta variável com a amamentação. Este é um resultado não esperado desde que crianças de baixo peso podem mostrar dificuldades de amamentação e o baixo peso ao nascer reflete as condições 
de saúde e nutrição materna, ficando, em sociedades desenvolvidas, abaixo de $5 \% .26$

Em suma não tendo havido diferença entre amamentar ou não e as variáveis estudadas, é possível levantar a hipótese de que isto ocorra devido ao fato de que as mães, na população estudada provavelmente, iniciam o processo de desmame ao preparar o filho para o ingresso na creche.

\section{Referências}

1. Moura EFA. Aleitamento ao seio. Freqüência de crianças amamentadas dentro do primeiro ano de vida. J Pediatr 1986; 61: 27-30

2. Souza LSF, Souza ELS, Barreto MRR, Ribeiro MR, Ramos RTT, Macedo JJB, Serra CR, Fernandes CE. Determinantes do êxito do aleitamento natural. J Pediatr 1991; 67: 42-50.

3. Procianoy RS. Factors affecting breastfeeding: the influence of cesarean section. J Trop Pediatr 1984; 30: 39-42.

4. Figueiredo LMH, Goulart EMA. Análise da eficácia do programa de incentivo ao aleitamento materno em um bairro periférico de Belo Horizonte (Brasil); 1980/ 1986/ 1992. J Pediatr (Rio de Janeiro) 1995; 71: 203-8.

5. Werck LN, Alpert JJ. Solid feeding guidelines. Lancet 1998; 352: 1569

6. WHO (World Health Organization). Innocenti declaration on the protection, promotion and support of breast-feeding. Ecol Food Nutr 1991; 26: 271-3

7. Andalaft RB, Gibbons AP, Padeiro RM, Ribeiro RP, Bueno LGS, Teruya KM, Murahovschi J. A influência do aleitamento materno no desenvolvimento neuropsicomotor. Rev Paul Pediatr 1999; 17: 20-4.

8. Ball TM, Wright AL. Health care costs of formula-feeding in the first year of life. Pediatrics 1999; 103: 870-6.

9. Wright AL, Bauer M, Naylor A, Sutcliffe E, Clark L. Increasing breastfeeding to reduce infant illness. Pediatrics 1998; 101: 837-43.

10. Puffer RR, Serrano CV. Características del peso al nacer. Washington, DC: Organización Panamericana de la Salud (OPS); 1988. (Publicación científica 504).

11. Berquó E, Souza JMP, Gotlieb SLD. Bioestatística. São Paulo: Epu; 1981.

12. Mc Cullagh P, Nelder JA. Generalized linear models. Boca Raton: Chapman \& Hall; 1998

13. Dean AG, Dean JÁ, Coulombier D, Brendel KA, Smith DC, Burton AH, Dicker RC, Sullivan K, Fagan RF, Arner TG. Epi Info. version 6: a word processing database, and statistics program for epidemiology on microcomputers [computer program]. Atlanta: Centers of Disease Control and Prevention; 1994.

14. Stata. Stata statistical software: release 6.0.College Station, Tx, Stata; 1999.
A curva de sobrevida para o aleitamento mostrando que mais de $80 \%$ das crianças foram amamentadas no primeiro mês de vida, embora com interrupção nos quatro primeiros meses para a metade delas, indica que a amamentação foi uma prática generalizada entre as mães.

15. Hosmer DW Jr, Lemeshow S. Applied survival analysis: regression modeling of time to event data. New York Wiley Interscience; 1999.

16. Amador M, Silva LC, Valdéz-Lazo F. Tendencias de la lactancia materna en Cuba y las Américas. Bol Ofic Sanit Panam 1994; 116: 204-11.

17. Rea MF. A amamentação e o uso do leite humano: o que recomenda a Academia Americana de Pediatria. J Pediatr (Rio de Janeiro) 1998; 74: 171-3.

18. Spinelli MGN. Prática alimentar de crianças menores de um ano de idade, atendidas em unidades básicas de saúde-escola [dissertação mestrado]. São Paulo: Faculdade de Saúde Pública da Universidade de São Paulo; 2000.

19. BEMFAM (Sociedade Civil Bem-Estar Familiar). Pesquisa nacional sobre demografia e saúde. Rio de Janeiro: Fundo das Nações Unidas para a Infância (UNICEF); 1997.

20. Costa COM, Figueiredo EM, Silva JB. Aleitamento materno: causas de desmame e justificativas para amamentar. J Pediatr (Rio de Janeiro) 1993; 69: 176-8.

21. Barber CM, Abernathy T, Steinmetz B, Charlebois J. Using a breastfeeding prevalence survey to identify a population for targeted programs. Can J Publ Health 1997; 88: 242-5.

22. Marchioni DML. Alimentação no primeiro ano de vida [dissertação mestrado]. São Paulo: Faculdade de Saúde Pública da Universidade de São Paulo; 1999

23. Candeias NMF. Educação em saúde na prevenção do risco de desmame precoce. Rev Saúde Pública 1983; 17: 7182.

24. Hammer LD, Bryson S, Agras WS. Development of feeding practices during the first 5 years of life. Arch Pediatr Adolesc Med 1999; 153: 189-94.

25. Sichieri R, Moura AS. Estudo dos fatores associados ao desmame precoce em crianças atendidas no Centro de Saúde de Várzea Paulista. J Pediatr (Rio de Janeiro) 1983; 55: 323-6.

26. Monteiro CA. O panorama da nutrição infantil nos anos 90 . Brasília, DF: UNICEF; 1996. p.1-17. (Cadernos de Políticas Sociais. Série Documentos para Discussão,1). 